\section{Western Blotting Method for the Immunostaining Detection of Glucuronides of Glycyrrhetic Acid Using Anti-Glycyrrhizin Monoclonal Antibody}

\author{
Shao-jie Shan, Hiroyuki TanaKa, and \\ Yukihiro SHOYAMA*
}

Department of Pharmacognosy, Faculty of Pharmaceutical Sciences, Kyushu University, 3-1-1 Maidashi, Higashi-ku, Fukuoka 8128582, Japan.

Received November 18, 1998; accepted December 18, 1998

A method for detecting glucuronides of glycyrrhetic acid using Western blotting was investigated. Glucuronides of glycyrrhetic acid separated by silica gel TLC were transferred to a polyvinylidene difluoride membrane. The membrane was treated with sodium periodate solution followed by bovine serum albumin, resulting in a glucuronides of glycyrrhetic acidBSA conjugate. Individual spots were stained by monoclonal antibody against glycyrrhizin. Immunostaining of glucuronides of glycyrrhetic acid was more sensitive compared to other staining methods. The newly established immunostaining method can be expanded to the distribution of glucuronides of glycyrrhetic acid in the plant body.

Key words Glycyrrhiza; Western blotting; glycyrrhizin; glycyrrhetic acid monoglucuronide; monoclonal antibody

Licorice (Glycyrrhiza spp.) is one of the most important Chinese medicines used in Chinese traditional medicine and is prescribed with other herbal medicines as a tussive, expectorant, and corrigent. ${ }^{1)}$ These pharmaceutical properties are due to glycyrrhizin (GC), which has antiprotein kinase, ${ }^{2)}$ antiulcer $^{3)}$ and antivira ${ }^{4)}$ activities, and is now used in Japan as an antiallergic ${ }^{5)}$ and hepatoprotective agent. ${ }^{6}$ In the recent rapid development of the molecular biosciences and their biotechnological applications, immunoassay systems using monoclonal antibodies (MAbs) against drugs and biologically active compounds of small molecular weight have become important tools ${ }^{7}$ for studies on receptor binding analyses, enzyme assays, and quantitative and/or qualitative analytical techniques in animals or plants due to their specific affinity. In previous papers we reported on the production of MAbs and enzyme immunoassays for solamargine, ${ }^{8)}$ forskolin, ${ }^{9,10)}$ opium alkaloids, ${ }^{11,12)}$ and marijuana compounds (cannabinoids). ${ }^{13)}$ More recently, we reported that an anti-GC MAb 5A8 prepared by us reacted specifically to GC, only weakly with a small number of structurally related GC, and did not react with other steroidal compounds. This indicates that the MAb has high specificity. ${ }^{14)}$ This paper describes the Western blotting of glucuronides of glycyrrhetic acid using the anti-GC MAb.

A culture medium containing the anti-GC MAb $5 \mathrm{~A} 8^{14)}$ was purified using a CHROMATOP Protein A column, ${ }^{15}$ ) dialyzed 5 times against water, and finally lyophilized to give IgG. Glucuronides of glycyrrhetic acid were applied to a TLC plate and developed with $n$-butanol-water- $\mathrm{AcOH}$ $(7: 2: 1)$.

The developed TLC plate was dried and then sprayed with a blotting solution mixture of isopropanol-methanol-water ( $1: 4: 20$ by volume). It was placed on a stainless steel plate, then covered with a polyvinylidene difluoride (PVDF) membrane sheet. After covering with a glass microfiber filter sheet, the whole was pressed evenly for $50 \mathrm{~s}$ with a $120^{\circ} \mathrm{C}$ iron as previously described ${ }^{16)}$ with some modification. The PVDF membrane was separated from the plate and dried. The blotted PVDF membrane was dipped in water containing $\mathrm{NaIO}_{4}(10 \mathrm{mg} / \mathrm{ml})$ with stirring at room temperature for $1 \mathrm{~h}$. After washing with water, carbonate buffer solution $50 \mathrm{~mm}$ $(\mathrm{pH}$ 9.6) containing bovine serum albumin (BSA) (1\%) was added, and stirred at room temperature for $3 \mathrm{~h}$. The PVDF membrane was washed with phosphate buffered saline (PBS) containing $0.05 \%$ of Tween 20 (T-PBS) twice for $5 \mathrm{~min}$, and then washed with water. The PVDF membrane was stained by the standard protocol for Western blotting ${ }^{17)}$ using MAb 5A8. The PVDF membrane was immersed in anti-GC MAb, and stirred at room temperature for $1 \mathrm{~h}$. After washing the PVDF membrane twice with TPBS and water, a 1000 -fold dilution of peroxidase-labeled goat anti-mouse IgG in GPBS (PBS containing $0.2 \%$ of gelatin) was added and stirred at room temperature for $1 \mathrm{~h}$. The PVDF membrane was washed twice with TPBS and water, and then exposed to 4-chloro-1naphthol- $0.03 \% \mathrm{H}_{2} \mathrm{O}_{2} 1 \mathrm{mg} / \mathrm{ml}$ in PBS solution, which was freshly prepared before use, for $10 \mathrm{~min}$ at room temperature. The reaction was stopped by washing with water. The immunostained PVDF membrane was then allowed to dry.

A separate PVDF membrane was placed on a glass microfiber filter sheet. A sliced Glycyrrhiza root was placed on the PVDF membrane, and they were pressed evenly for $6 \mathrm{~h}$. The blotted PVDF membrane was dipped in water containing
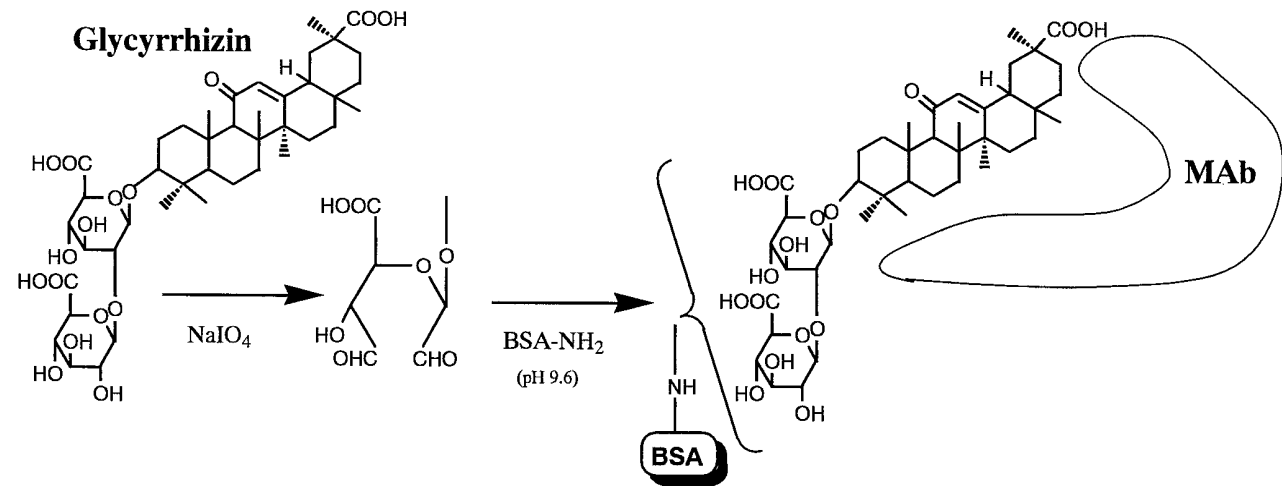

Fig. 1. Schematic Evidence for Western Blotting of GC on PVDF Membrane Using Anti-GC MAb 
A

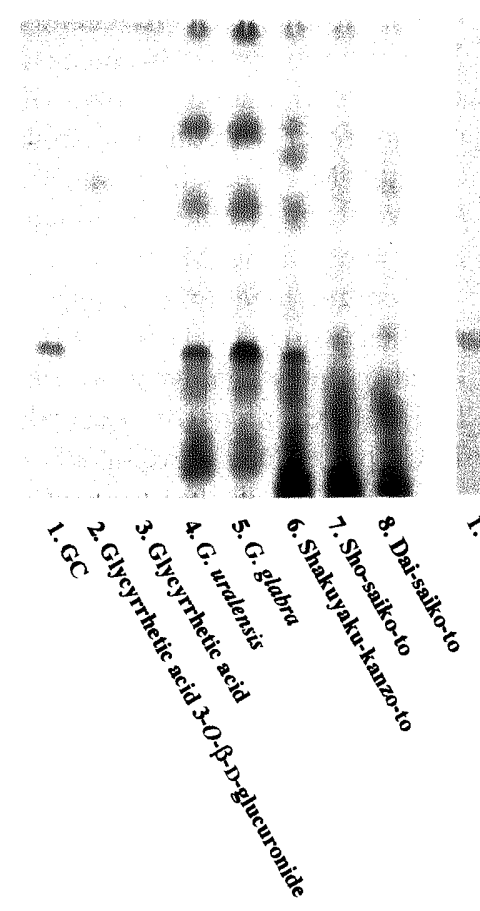

B

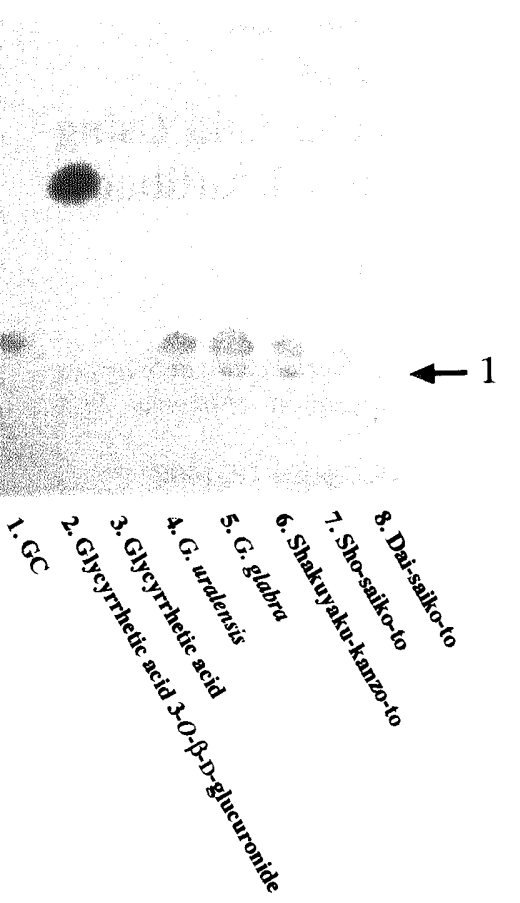

Fig. 2. $\mathrm{H}_{2} \mathrm{SO}_{4}$ Staining on TLC (A) and Western Blotting of GC, Related Compounds, Glycyrrhiza Species, and Kampo Prescriptions on PVDF Membrane (B)

Glycyrrhetic acid and its glucuronides were applied to TLC plates and developed with $n$-butanol-water-AcOH $(7: 2: 1)$. One developed TLC plate was sprayed with $\mathrm{H}_{2} \mathrm{SO}_{4}$ and heated to stain (A). Another TLC plate was blotted on the PVDF membrane by heating. The blotted PVDF membrane was dipped in water containing $\mathrm{NaIO}_{4}$ for $1 \mathrm{~h}$. After washing with water, carbonate buffer solution $50 \mathrm{~mm}$ containing BSA was added and stirred for $3 \mathrm{~h}$. The PVDF membrane was stained by standard protocols for Western blotting using MAb 5A8 (B). Lane 1: GC (2 $\mu \mathrm{g})$; lane 2: glycyrrhetic acid 3-O- $\beta$-D-glucuronide ( $2 \mu \mathrm{g})$; lane 3: glycyrrhetic acid ( $2 \mu \mathrm{g})$; lane 4: G. uralensis; lane 5: G. glabra; lane 6: Shakuyakukanzo-to; lane 7: Sho-saiko-to; lane 8: Dai-saiko-to.

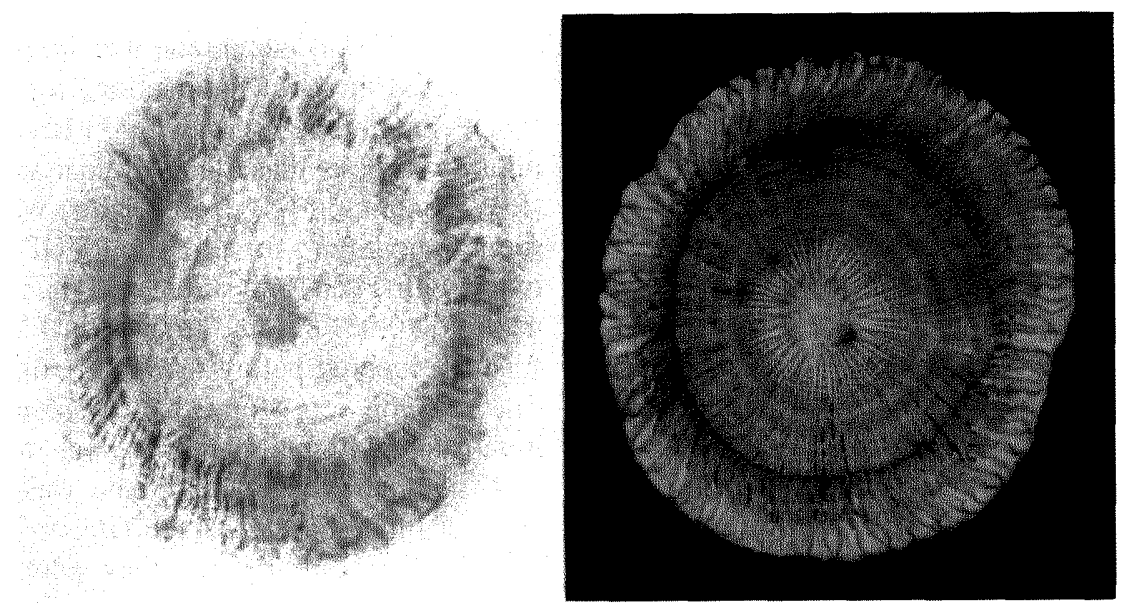

Fig. 3. Immunocytolocalization of GC Using Anti-GC MAb in Glycyrrhiza Root

$\mathrm{NaIO}_{4}$ for $1 \mathrm{~h}$. After washing with water, carbonate buffer solution $50 \mathrm{~mm}$ containing BSA was added, and stirred for $3 \mathrm{~h}$. The PVDF membrane was stained by the same method as indicated above using MAb 5A8.

Direct detection by immunostaining of small molecular compounds on TLC plates is impossible. Moreover, since GC and its related compounds on PVDF membranes are washed out by buffer solution or water without fixing, we devised a new method which separates the two functions of GC into that of an epitope against a MAb and that of conjugating with the membrane using a simple and well-known chemical reaction on the membrane, as indicated in Fig. 1. Therefore we established that GC was blotted on the PVDF membrane from the developed TLC plate and conjugated with protein after cleavage of the sugar moiety by $\mathrm{NaIO}_{4}$, as previously reported. ${ }^{18)}$ The PVDF membrane incubated in the absence of $\mathrm{NaIO}_{4}$ is essentially free of staining for GC (data not shown). Therefore the conjugation step between the sugar moiety and protein is necessary for Western blotting. The antigen synthesized by $\mathrm{NaIO}_{4}$ cleavage via conjugation with the carrier protein is necessary for this new Western blotting method, because when different types of hapten-carrier protein conjugate are used, Western blotting cannot be detect them without it. ${ }^{19)}$

Figure 2 shows $\mathrm{H}_{2} \mathrm{SO}_{4}$ staining (A) and Western blotting (B) of GC and various samples. Lanes 1 to 3 show the bands 
of GC, glycyrrhetic acid monoglucuronide, and glycyrrhetic acid, respectively. The Western blotting method was considerably more sensitive than $\mathrm{H}_{2} \mathrm{SO}_{4}$ staining. In addition, it is suggested that the specific reactivity of the sugar moiety in $\mathrm{GC}$ against $\mathrm{MAb}$ may be modified by $\mathrm{NaIO}_{4}$ treatment of GC on the membrane, resulting in the detection of glycyrrhetic acid monoglucuronide, which as previously reported ${ }^{14)}$ has a small cross-reactivity ( $4.36 \%$ ), by Western blotting (Fig. 2-B, lane 2). The greater sensitivity of glycyrrhetic acid monoglucuronide compared with GC is due to their blotting efficiencies. GC is strongly bound to the TLC plate due to three carboxylic acid groups in a molecule. Although $\mathrm{H}_{2} \mathrm{SO}_{4}$ staining (Fig. 2-A) detected many spots, probably including sugars and different types of saponins (Fig. 2-A, lanes 4 and 5), in two Glycyrrhiza samples, Western blotting (Fig. 2-B) only detected GC and a small amount of unknown compounds (arrow 1). A GC aglycone, glycyrrhetic acid (arrow) was not detected by Western blotting, indicating that this new Western blotting method requires a sugar moiety, as discussed above. Two prescriptions containing licorice, Shakuyakukanzo-to (lane 6) and Sho-saiko-to (lane 7), clearly showed a GC band. However, Dai-saiko-to which does not contain licorice was not stained by Western blotting, as shown in Fig. 2-B, lane 8 .

As another application of this methodology, the immunocytolocalization of GC in Glycyrrhiza organs was investigated. Figure 3 illustrates the immunocytolocalization of GC in fresh Glycyrrhiza inflata root. The cortex contains a higher concentration of GC than the xylem, indicating a good agreement with a previous report. ${ }^{20)}$

We previously developed an ELISA for GC that is 1000fold more sensitive than HPLC. ${ }^{14)}$ Therefore a combination of the new Western blotting method and ELISA can be used to survey natural resources of GC and to analyze concentrations of GC and related compounds in drugs and/or animal plasma samples rapidly and simply. We detected an unknown compound in two Glycyrrhiza species and one Kampo pre- scription, suggesting it possesses the glycyrrhetic acid framework in a molecule although it has not been isolated yet. This methodology can theoretically be expanded for various metabolic glucuronides and for all sugar conjugates.

\section{REFERENCES AND NOTES}

1) Japanese Pharmacopoeia (13th edition), pp. 1140-1141 (1996).

2) Ohtsuki K., Ishida N., Biochem. Biophys. Res. Commun., 157, 597604 (1988).

3) Doll R., Hill I. D., Hutton C., Underwood D. L., Lancet, ii, 793-796 (1962).

4) Pompei R., Flore O., Marccialis M. A., Pani A., Loddo B., Nature, 281, 689-690 (1979).

5) Kuroyanagi T., Saito M., Jpn. J. Allerg., 15, 67-74 (1966).

6) Fujita H., Sakurai T., Toyoshima S., Pharmacometrics, 16, 637-645 (1978).

7) Weiler E. W., Chemistry of Plant Production, Springer, Berlin, 1990, pp. $145-220$.

8) Ishiyama M., Shoyama Y., Murakami H., Shinohara H., Cytotechnology, 18, 153-158 (1996)

9) Sakata R., Shoyama Y., Murakami H., Cytotechnology, 16, 101-108 (1994).

10) Yanagihara H., Sakata R., Shoyama Y., Murakami H., Planta Med., 62, $169-172(1996)$

11) Shoyama Y., Fukada T., Murakami H., Cytotechnology, 19, 55-61 (1995).

12) Shoyama Y., Kawachi F., Tanaka H., Nakai R., Shibata T., Nishi K., Forensic Sci. Int., 91, 207-217 (1998).

13) Tanaka H., Goto Y., Shoyama Y., J. Immunoassay, 17, 321-342 (1996).

14) Tanaka H., Shoyama Y., Biol. Pharm. Bull., submitted.

15) Langone J. J., J. Immunol. Methods, 51, 33-45 (1982).

16) Taki K., Kasama T., Handa S., Ishikawa D., Anal. Biochem., 223, $232-238$ (1994).

17) Towbin H., Staehelin T., Gordon J., Proc. Natl. Acad. Sci. U.S.A., 76, $4350-4354$ (1979).

18) McLean I. W., Nakane P. K., J. Histochem. Cytochem., 22, 1077 1083 (1974).

19) Xuan L. J., Tanaka H., Xu Y. M., Shoyama Y., Cytotechnology, in press.

20) Hayashi H., Fukui H, Tabata M., Planta Med., 59, 351 1 353 (1993). 